\title{
APLICABILIDADE DO ALGORITMO DE LEVENBERG-MARQUARDT PARA ANÁLISE DE GERAÇÃO DE ENERGIA ELÉTRICA DE UM SISTEMA FOTOVOLTAICO
}

\section{APPLICABILITY OF LEVENBERG-MARQUARDT ALGORITHM FOR POWER GENERATION ANALYSIS OF THE SYSTEM PHOTOVOLTAIC}

\author{
Elisângela Pinheiro*E-mail: elisangelapinheirors@gmail.com \\ Ricardo Rüther*E-mail: ruther@mbox.ufsc.br \\ Adalberto Lovato ** E-mail: proflovatosr@gmail.com \\ * Universidade Federal de Santa Catarina, Florianópolis (UFSC), Florianópolis, SC \\ ${ }^{* *}$ Faculdade Horizontina, Horizontina/ RS
}

\begin{abstract}
Resumo: Este trabalho avalia o uso de redes neurais artificiais para análise de geração de eletricidade para um sistema fotovoltaico conectado a rede. Inicialmente, são descritas as características e potencial matemático de tratamento de dados apresentados pelas redes. Em seguida, são testadas várias configurações com o objetivo de buscar a mais adequada ao caso. Foram utilizados dados de 2014 da geração de energia, de temperatura ambiente, de temperatura dos módulos da incidência de radiação solar e da hora do dia. A aplicação utilizou os algoritmos de Levenberg-Marquardt para obtenção dos parâmetros da rede e o critério do erro médio quadrado para medir o desempenho. O treinamento foi reealizado com 5, 10,15, 20, 25, 30 e 60 neurônios na camada oculta. As funções de transferência foram logsigmoide e purelin. O melhor resultado foi realizado foi obtido com 25 neurônios, com coeficiente de correlação de 0,98.
\end{abstract}

Palavras-chave: Energia solar fotovoltaica. Redes Neurais Artificiais. Algoritmo LevembergMarquardt. Função de transferência Logsigmóide. Função de transferência purelin.

Abstract: This paper talk about the applicability of artificial neural networks for power generation analysis for a photovoltaic system connected to the grid. First, the characteristics and mathematical potential of data processing presented by networks are described. Then, several configurations are tested in order to find the most appropriate one. Data from 2014 were used for energy generation, room temperature, module temperature, solar radiation incidence and time of day. The application used the Levenberg-Marquardt algorithms to obtain the network parameters and the criterion of the mean square error to measure the performance. The training was reealized with $5,10,15,20,25,30$ and 60 neurons in the hidden layer. The transfer functions were logsigmoid and purelin. The best result was obtained with 25 neurons, with a correlation coefficient of 0.98 .

Keywords: Photovoltaic Solar Energy. Artificial Neural Networks. Levemberg- Marquardt Algorithm. Transfer function logsigmoide. Transfer function purelin.

\section{INTRODUÇÃO}

Atualmente, o fornecimento de energia é um dos grandes problemas mundiais. Seu consumo é crescente, e, o desafio energético é satisfazer está crescente demanda de energia, proteger as fontes usualmente utilizadas, buscar a 
exploração por fontes renováveis e, ao mesmo tempo, confrontar a ameaça das mudanças climáticas e redução de emissão de poluentes atmosféricos.

Com a evolução tecnológica de sistemas fotovoltaicos, com células cada vez mais eficientes, conforme apresenta NREL (2016), surgem desafios em áreas complementares, na previsão de incidência de irradiação em um instante futuro, e na avaliação de desempenho de sistemas fotovoltaicos, como é o caso deste estudo.

Essa avaliação é fundamental para determinar o potencial de energia solar fotovoltaica, auxiliar na administração do despacho de energia e analisar a qualidade da potência obtida.

Uma das formas mais comuns de avaliação de desempenho de energia solar é a utilização de softwares, métodos computacionais e algoritmos de computação inteligente, como as redes neurais artificiais (RNA) que buscam obter as predições de incidência de radiação e analisar a geração de energias necessárias (GILMAN, DOBOS, \& KASBERG, 2012).

Neste contexto, este estudo se propõe a utilizar os algoritmos de LevenbergMarquart, para avaliar a geração de eletricidade de um sistema fotovoltaico conectado à rede (SFVCR) e integrado a uma edificação instalado no Brasil, que se encontra em operação desde setembro de 1997, localizado no prédio de Engenharia Mecânica da Universidade Federal de Santa Catarina (UFSC) em Florianópolis (RÜTHER, 1998; RÜTHER e DACOREGIO, 2000). Por meio deste estudo, busca-se analisar o uso de redes neurais artificiais para avaliar o desempenho de um sistema fotovoltaico conectado à rede, objetivando apurar se o algoritmo é adequado para este tipo de avaliação.

As contribuições deste artigo serão as seguintes:

a. Avaliar se o algoritmo Levenberg-Marquart de redes neurais é adequado para avaliação de potência gerada por um sistema fotovoltaico conectado à rede.

b. Avaliar se a análise de geração de eletricidade de sistema fotovoltaico conectado à rede utilizando dados de um ano é representativa.

c. Testar a rede que obter o melhor coeficiente de desempenho para comparar os dados de potência medidos no sistema fotovoltaico, em relação aos dados de potência previstos pela rede. 
Este artigo está organizado em seis seções: a seção 1 apresenta a importância da utilização de redes neurais na área de energia solar; na seção 2 são discutidos trabalhos relacionados sobre utilização de modelos de redes neurais, contendo uma descrição de como as redes contribuíram na área de geração de energia solar; na seção 3, é apresentada a metodologia utilizada no trabalho; a seção 4, descreve o desenvolvimento das redes e como elas foram testadas de acordo com o algoritmo proposto; a seção 5 apresenta os resultados de qual rede é mais adequado para o caso e a seção 6 apresenta uma breve conclusão do trabalho.

\section{TRABALHOS RELACIONADOS}

Os métodos estatísticos computacionais de redes neurais são muito utilizados, pois permitem avaliar o desempenho de sistemas fotovoltaicos (MEKKI et al., 2007), bem como, dimensionar sistemas fotovoltaicos, realizar levantamentos de disponibilidades do recursos solar (FIORIN et al., 2011). Para Mellit et al., (2009) a vantagem de usar uma base de redes neurais em sistemas fotovoltaicos é que a mesma proporciona modelos confiáveis, para estudos de incidência de irradiação, principalmente em áreas isoladas, onde os dados meteorológicos nem sempre são disponíveis, bem como, para a resolução de problemas desde modelagens, simulação e controle destes sistemas.

Alguns dos estudos relacionados com a aplicação de redes neurais artificiais na área de energia solar fotovoltaica foi abordado por Mellit e Kalogirou (2008) e Mellit e Kalogirou (2011), que apresentam uma revisão das principais aplicações e das mais variadas configurações de redes, aplicadas para a previsão de radiação solar.

Estudos em um sistema integrado à edificação e conectado à rede foi realizado em Hon Kong, com a finalidade de verificar o desempenho de uma previsão de curto prazo em tempo real (CHOW et al., 2012). Fiorin et al., (2011) realizou um comparativo de desempenho de diferentes algoritmos de treinamento (Levenberg-Marquardt, Resilient Backpropagation, Gradiente conjugado escalar, Taxa de aprendizagem variável e Taxa de aprendizagem variável com momento) para a análise do parâmetro de radiação solar para São Martinho da Serra - RS. 
$\mathrm{Na}$ Índia, os pesquisadores Yadav e Chandel, (2012) utilizaram o método Levenberg-Marquardt para a predição de radiação solar. Na Grécia, Chatziagorakis et.al. (2014), também utilizaram o Levenberg-Marquardt para melhorar a gestão de energia através da irradiação solar presente.

Os estudos realizados até o momento, que utilizaram métodos e algoritmos de redes neurais artificiais, são mais aplicados em relação ao dimensionamento de sistemas fotovoltaicos para a predição de incidência de radiação, mas, nenhum deles pesquisou se as redes neurais são adequadas para analisar a geração de energia de um sistema fotovoltaico integrado na edificação e conectado à rede, conforme proposta deste artigo.

\section{MÉTODOS}

A RNA foi estruturada com dados de desempenho elétrico, temperatura ambiente, temperatura do módulo, irradiância e intervalo de tempo. Sensores de irradiância eram capazes de medir a irradiância global e a total - horizontal e inclinada. O intervalo de tempo entre os registros foi de 5 minutos. O sistema fotovoltaico é conectado à rede com potência nominal de $2 \mathrm{kWp}$. Os módulos são de filme fino de silício amorfo hidrogenado (a-Si:H), de dupla junção.

Primeiramente, os dados foram normalizados e depurados, a seguir foram determinadas as variáveis de entrada da rede neural: irradiância, temperatura do módulo, temperatura ambiente e a hora do dia - a cada cinco minutos, desde 07h00min até $18 \mathrm{~h} 00 \mathrm{~min}$. A saída da rede — target — foi a energia gerada.

Foi utilizado o algoritmo Levenberg-Marquart, conforme apresentado por Bousfield (2008), é o método mais adequado para este tipo de estudo.

Quanto à arquitetura da rede foram utilizados os parâmetros apresentados no Quadro 1. 
Quadro 1 - Arquitetura da RNA: critérios e quantitativos definidos

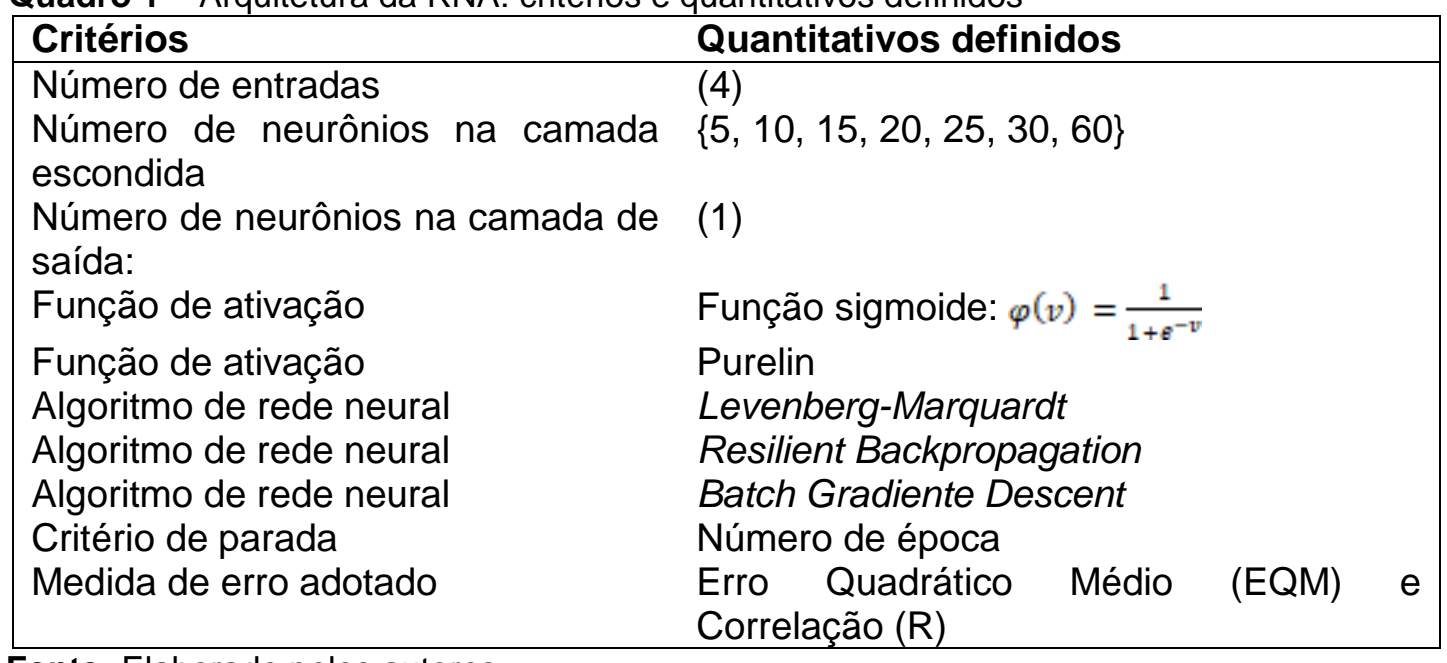

Fonte: Elaborado pelos autores

A arquitetura da RNA tem quatro dados de entrada, sendo: irradiação, temperatura ambiente, temperatura do módulo e tempo. Na camada escondida foram testados sete quantitativos de neurônios de 5 até 60 . Na camada de saída, apenas um neurônio. Como função de ativação, para os neurônios na camada escondida foi utilizada a função sigmóide e para a camada de saída temos a função de ativação purelin. Como critério de parada do treinamento foram utilizados o número de épocas, o valor do Erro Quadrático Médio e o valor do coeficiente de Correlação.

Para processar o treinamento foi utilizado o software MATLAB versão R2015b, License Student.

A Figura 1 mostra a sequência metodológica do estudo. Primeiramente, foi realizada a seleção de dados de entrada e saída da rede. No segundo passo, foi configurada a rede, a configuração do treinamento e os testes das redes. E por último, aconteceu o treinamento das diversas redes, seguido da seleção da configuração que apresentou melhor resultado. 


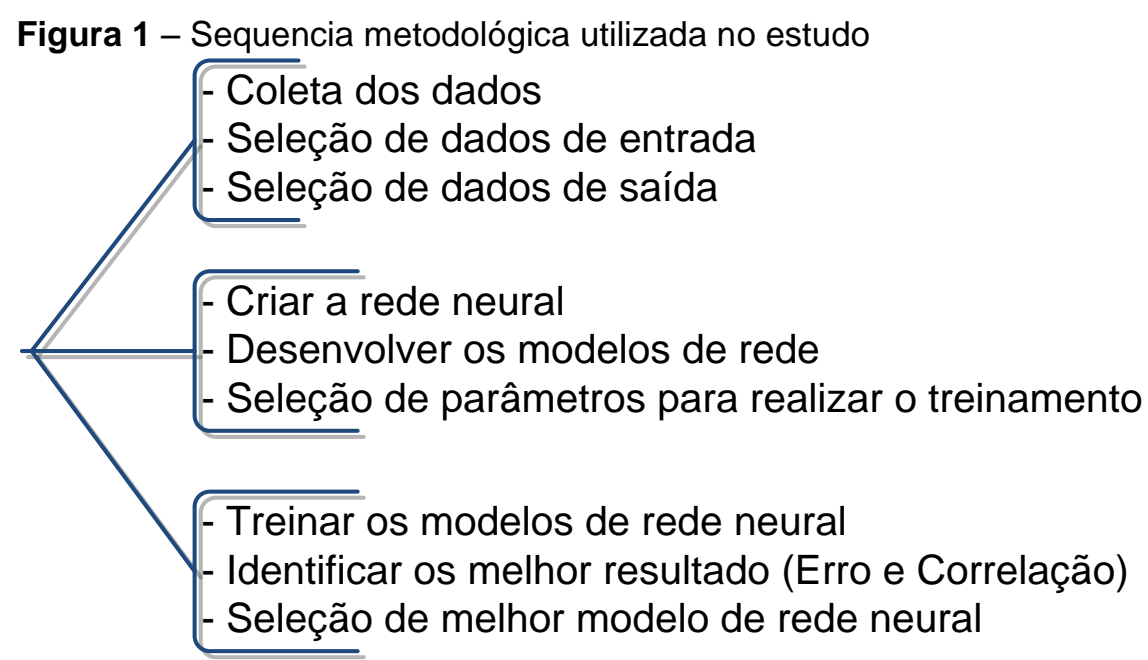

\section{DESENVOLVIMENTO}

No desenvolvimento, primeiramente foram coletados 38.266 registros meteorológicos referentes ao ano de 2014 da estação solarimétrica instalada junto ao sistema fotovoltaico. Como dados de entrada da rede, foram utilizados, irradiância incidente no mesmo plano inclinado que o dos módulos fotovoltaicos, temperatura ambiente, temperatura do módulo, e horário da coleta. Como dados de saída, ou Target, foi utilizado dados de potência gerada pelo sistema fotovoltaico.

Para testar a rede foram utilizados dados referentes aos meses da estação da primavera do ano de 2014.

A Tabela 1 apresenta os valores máximos e mínimos dos dados de entrada e saída (target).

Tabela 1 - Valores de dados de entrada e saída utilizadas na estrutura das redes

\begin{tabular}{llllll}
\hline & \multicolumn{3}{c}{ Valores de entrada } & \multicolumn{2}{c}{ Valores de saída } \\
& Irradiação & $\begin{array}{c}\text { Temperatura } \\
\text { ambiente }\end{array}$ & $\begin{array}{l}\text { Temperatura } \\
\text { do módulo }\end{array}$ & Horário & Potência \\
\hline Máximo & 1317,86 & 40,99 & 62,86 & 18,00 & 1914,25 \\
Mínimo & 1,73 & 13,86 & 14,87 & 7,00 & 0,04 \\
\hline
\end{tabular}

Fonte: Elaborado pelos autores

Estes dados foram depurados antes de serem importados para o software MATLAB.

A Figura 2 mostra a rede utilizada neste artigo, o modelo de rede neural apresenta quatro dados de entrada, uma camada escondida, com neurônios de ativação e uma camada de saída. 
Figura 2 - Estrutura da rede neural artificial do construída no estudo.

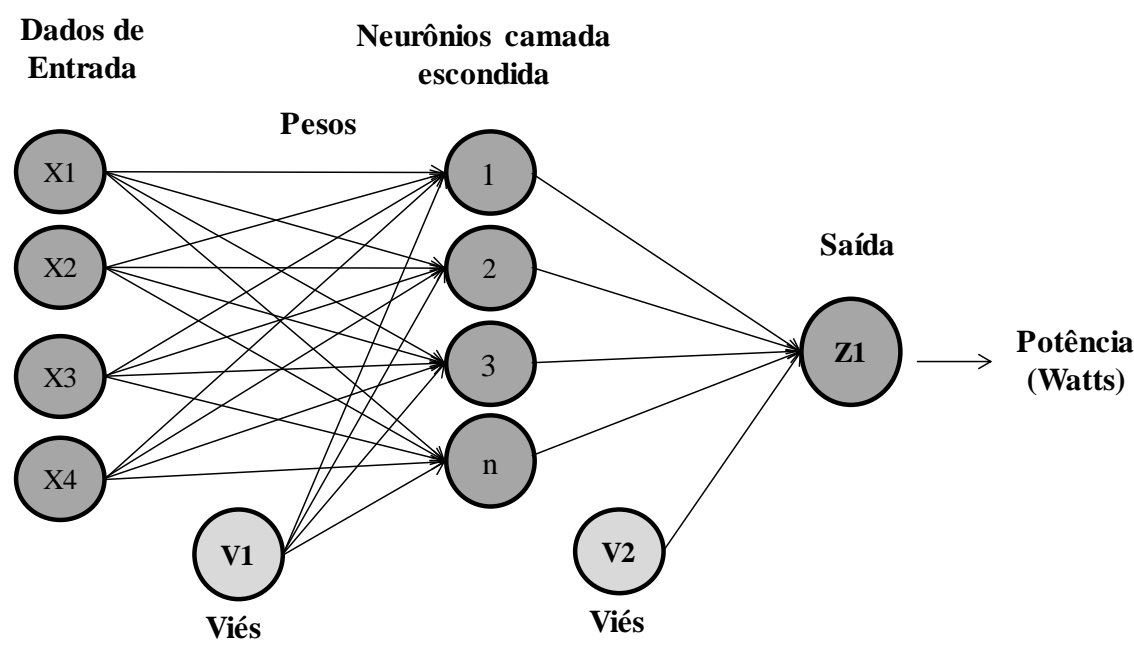

Como função de transferência neural na camada oculta a função Log Sigmóide e na camada de saída purelin. Estas funções são responsáveis pela realização dos cálculos a partir das variáveis de entrada. Foram realizados testes com 5, 10, 15, 20, 30 e 60 neurônios para cada um dos algoritmos.

A Figura 3 apresenta o gráfico da função Log Sigmóide representada pela letra a e a letra b mostra o gráfico da função purelin.

Figura 3 - Função Log Sigmoide e Função Purelin

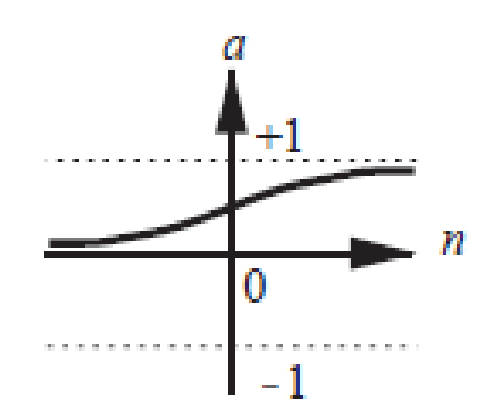

$$
a=\log \operatorname{sig}(n)
$$

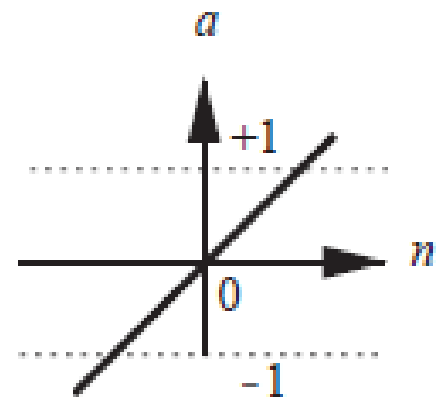

$a=\operatorname{purelin}(n)$

Letra a) função Log Sigmóide Letra b) função Purelin

Fonte: Adaptado Hagan, (2015)

O processo de construção e treinamento da rede seguiu os seguintes passos:

$1^{\circ}$. tratar os dados de entrada

$2^{2}$. definir o número de neurônios

3․ definir o tipo de treinamento e validação da rede.

$4^{\circ}$. desenvolver a rede e treinar a rede 
5․ gerar valores de saída

6‥ analisar os dados de saída

7‥ comparar os dados de saída da rede com valores desejados.

8‥ Caso não tenha obtido dados satisfatórios retornar a etapa inicial.

A Tabela 2 mostra um dia da estação da primavera que foi sorteado aleatoriamente. Estes valores foram utilizados para fazer a simulação e para testar a rede neural.

Tabela 2 - Valores de dados de entrada e saída utilizadas na estrutura das redes

\begin{tabular}{lrrrrrc}
\multicolumn{2}{l}{$\begin{array}{l}\text { Valores Sorteados } \\
\text { Data }\end{array}$} & $\begin{array}{c}\text { Irradiância } \\
\text { W/m } \mathbf{m}^{2}\end{array}$ & $\begin{array}{c}\text { Temperatura } \\
\text { ambiente } \\
\text { Co }\end{array}$ & $\begin{array}{c}\text { Temperatura } \\
\text { do módulo } \\
\text { Coo }\end{array}$ & $\begin{array}{c}\text { Hora } \\
\text { do dia }\end{array}$ & $\begin{array}{c}\text { Potência } \\
\text { target } \\
\text { W }\end{array}$ \\
\hline & $05 / 11 / 14$ & 181,92 & 25,4 & 27,57 & $7: 50$ & 75,31 \\
& $05 / 11 / 14$ & 190,73 & 25,8 & 29,2 & $8: 00$ & 252,85 \\
& $05 / 11 / 14$ & 109,31 & 22,51 & 25,22 & $9: 00$ & 194,31 \\
& $05 / 11 / 14$ & 313,27 & 22,37 & 34 & $10: 00$ & 453,19 \\
NOV & $05 / 11 / 14$ & 481,65 & 23,3 & 36,43 & $11: 00$ & 706,28 \\
Primavera & $05 / 11 / 14$ & 1011,43 & 29,45 & 41,76 & $12: 00$ & 1562,09 \\
& $05 / 11 / 14$ & 988,45 & 29,31 & 49,84 & $13: 00$ & 1531,05 \\
& $05 / 11 / 14$ & 988,07 & 31,82 & 46,54 & $14: 00$ & 1464,87 \\
& $05 / 11 / 14$ & 832,56 & 29,6 & 48,26 & $15: 00$ & 1191,59 \\
& $05 / 11 / 14$ & 580 & 29,73 & 41,11 & $16: 00$ & 752,27 \\
& $05 / 11 / 14$ & 98,97 & 26,43 & 30,27 & $17: 00$ & 154,03 \\
& $05 / 11 / 14$ & 28,33 & 24,98 & 26,66 & $18: 00$ & 36,72 \\
\hline
\end{tabular}

Fonte: Elaborado pelos autores

\section{RESULTADOS}

Nesta seção estão apresentados os resultados obtidos. Para testar as redes, foram utilizados diversos treinamentos para cada rede estruturada, com seus devidos pesos, conforme apresentado no desenvolvimento.

No treinamento as sete redes apresentaram um coeficiente superior a 0,98, sendo este uma forte correlação. E todas as redes são consideradas ótimas conforme valores obtidos de MSE e R. Mas ressalva-se a importância de quanto mais diferenciado for o número de neurônios, melhor para fazer a escolha ou uso de um número de neurônio mais adequado para otimizar o funcionamento da rede. Neste estudo, a rede selecionada foi a de 25 neurônios conforme mostra a Tabela 3 , essa rede foi a que apresentou o menor erro. 
Tabela 3 - Valores dos resultados de MSE e $\mathrm{R}$ das redes

\begin{tabular}{cccccccc}
\hline Neurônios & EQM (MSE) & Época & \multicolumn{4}{c}{ Correlação (R) } \\
& & & Treinamento & Validação & Teste & Todos \\
\hline $\mathbf{5}$ & 4233,02 & 165 & 0,98949 & 0,98859 & 0,98873 & 0,98924 \\
10 & 4331,55 & 60 & 0,98855 & 0,98843 & 0,98801 & 0,98845 \\
15 & 4420,37 & 47 & 0,98845 & 0,9881 & 0,98902 & 0,98848 \\
20 & 4237,29 & 509 & 0,98819 & 0,98889 & 0,98758 & 0,98821 \\
$\mathbf{2 5}$ & $\mathbf{3 7 5 0 , 4 3}$ & $\mathbf{2 0 4}$ & $\mathbf{0 , 9 8 9 6 2}$ & $\mathbf{0 , 9 9 0 0 5}$ & $\mathbf{0 , 9 9 0 4}$ & $\mathbf{0 , 9 8 9 8 1}$ \\
30 & 4465,74 & 58 & 0,9892 & 0,98831 & 0,99049 & 0,98925 \\
60 & 4959,30 & 56 & 0,98876 & 0,98719 & 0,98845 & 0,98847
\end{tabular}

Fonte: Elaborado pelos autores

Na Figura 4 mostra a curva de valores do Mean Square Error (MSE) da RNA, visto que o valor do coeficiente de correlação e o MSE obteve melhor resultado para a rede neural com 25 neurônios da camada escondida.

Figura 4- Erro Quadrático Médio (MSE)

Melhor desempenho de validação é 3750,4344 na época 198

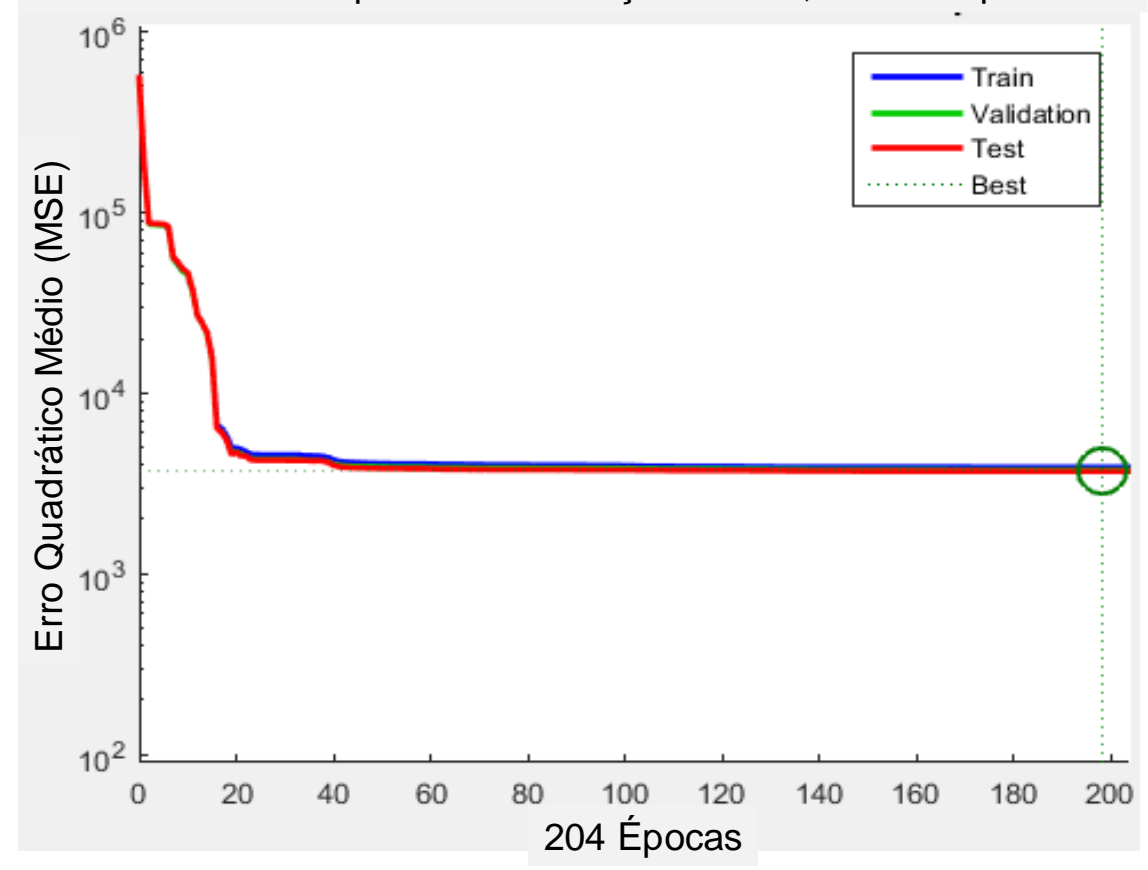

As épocas apresentadas pela rede neural, por definição utilizam todos os dados disponíveis. Do total dos dados, ele considera $75 \%$ dos dados para treinar a rede. Então, percorre grupos de 25 dados e atualiza os parâmetros da rede. Os 
grupos são formados de acordo com o número de neurônios; desta forma, avaliando o resultado do gráfico.

A Figura 5 mostra o gráfico de desempenho da rede neural. Para avaliar 0 desempenho da rede do algoritmo Levenberg-Marquardt temos o gradiente, o mu (valor adaptativo para auxiliar no cálculo do desempenho da rede) e a verificação de validação.

O gradiente mostra o grau de inclinação da rede na superfície do erro quando busca alcançar o erro mínimo global. Portanto, em cada etapa do treinamento, o vetor de peso utilizado anteriormente é alterado com objetivo de provocar uma brusca queda na superfície do erro. Observa-se que quando a inclinação do gradiente diminui, aumenta a distância do erro e vice-versa.

O Mu é um incremento adicionado para se chegar ao resultado final, enquanto que o gradiente procura obter o menor valor de erro. Portanto, à medida que o gradiente diminui, o Mu aumenta. Como o Mu é um incremento que acontece para se chegar ao mínimo valor, o MSE busca se aproximar do valor mínimo do erro.

Durante a verificação de validação, indica que foram realizadas 6 iterações consecutivas e o resultado não sofreu mais alteração, com isso, o teste é finalizado, conforme destacado com um círculo nas 6 últimas iterações.

Figura 5- Desempenho da rede neural com 25 neurônios
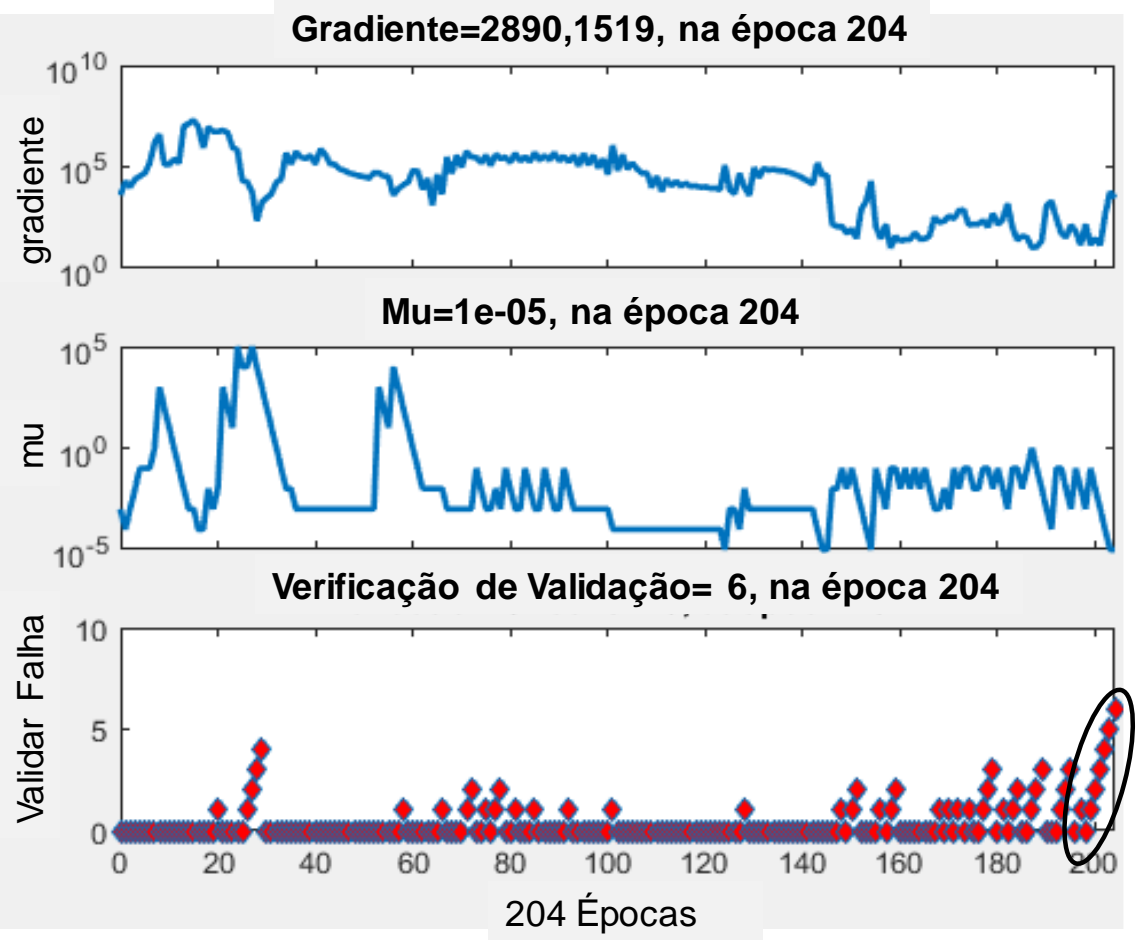

Revista Produção Online. Florianópolis, SC, v.17, n. 4, p. 1204-1217, 2017. 
Para o treinamento das redes neurais, os dados foram divididos em três subconjuntos, sendo que o primeiro subconjunto utiliza $70 \%$ do total dos dados selecionados aleatoriamente para calcular o gradiente e realizar o ajuste dos pesos da rede. Para validar os dados, é utilizado um subconjunto de $15 \%$ do todas dos dados também selecionado aleatoriamente, mostrando os erros da rede, podendo ser reduzido durante o treinamento e aumentando à medida que o treinamento ocorre. Para testar a rede, é utilizado o terceiro subconjunto de $15 \%$ do total dos dados, para testar a efetividade da rede e também para verificar se o teste chegou no erro encontrado na validação com um número de iterações significativamente diferentes, que dá indícios de uma divisão de dados não efetiva. Caso isso ocorra, a rede é testada com uma nova divisão dos subconjuntos dos dados.

A Figura 6 mostra o valor do coeficiente de correlação $(R)$ dos dados da rede, quanto mais próximos de 1 forem os valores, melhor é a correlação dos dados, ou seja, quão próximo estão os dados dos valores reais.

Figura 6 - Regressão da rede neural com 25 neurônios da camada escondida

Treinamento: $\mathbf{R}=\mathbf{0 , 9 8 9 6 2}$

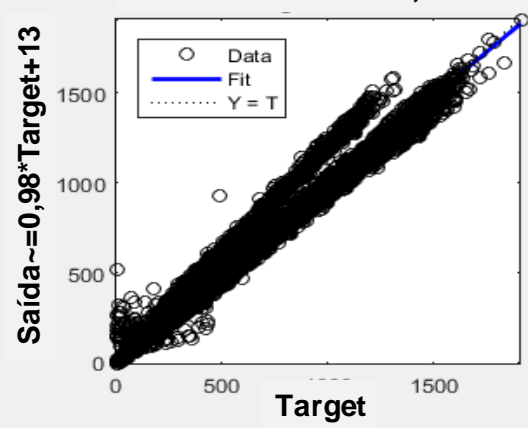

Teste: R=0,9904

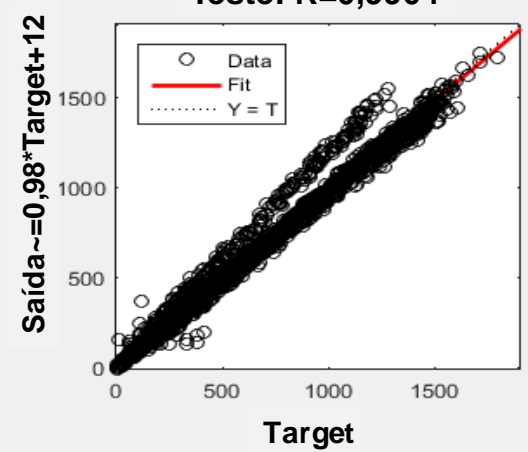

Validação: $\mathbf{R}=0,99005$

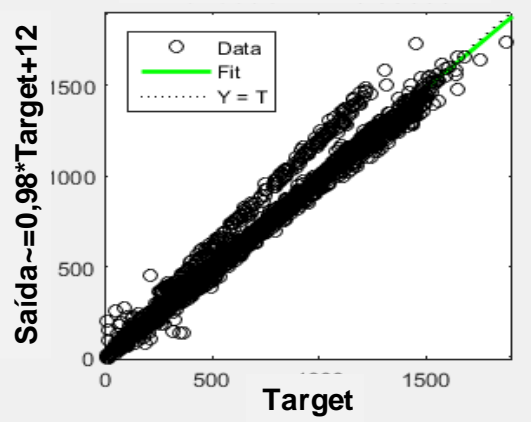

Todos: $\mathbf{R}=\mathbf{0 , 9 8 9 8 1}$

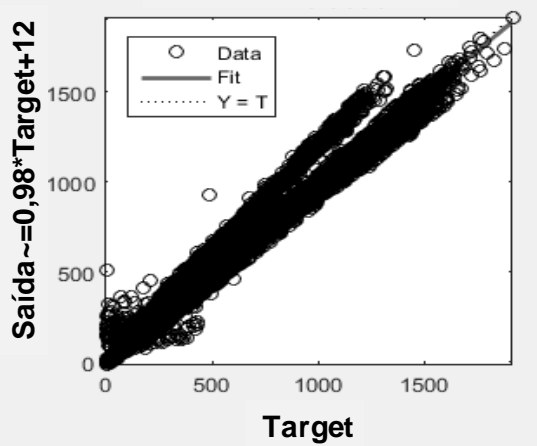

A linha pontilhada que atravessa no gráfico, representa a saída desejada a ser atingida e a linha contínua colorida mostra a melhor regressão obtida no ajuste linear entre as saídas desejadas e as saídas reais. 
A Tabela 4 mostra valores de simulação conforme os valores sorteados.

Tabela 4 - Valores de simulações das redes

\begin{tabular}{|c|c|c|c|c|c|c|c|}
\hline \multicolumn{2}{|c|}{$\begin{array}{c}\text { Valores Sorteados } \\
\text { Data }\end{array}$} & \multirow{2}{*}{$\begin{array}{c}\text { Irradiância } \\
181,92\end{array}$} & \multirow{2}{*}{$\begin{array}{c}\text { Temp.Amb. } \\
25,4\end{array}$} & \multirow{2}{*}{$\begin{array}{c}\begin{array}{c}\text { Temp.do } \\
\text { Mód }\end{array} \\
27,57\end{array}$} & \multirow{2}{*}{$\begin{array}{l}\text { Time } \\
7: 50\end{array}$} & \multirow{2}{*}{$\begin{array}{c}\begin{array}{c}\text { Pot } \\
\text { Target }\end{array} \\
75,31\end{array}$} & \multirow{2}{*}{$\begin{array}{c}\begin{array}{c}\text { Pot } \\
\text { Simulada }\end{array} \\
216.39\end{array}$} \\
\hline \multirow{12}{*}{$\begin{array}{c}\text { NOV } \\
\text { Primavera }\end{array}$} & $05 / 11 / 14$ & & & & & & \\
\hline & $05 / 11 / 14$ & 190,73 & 25,8 & 29,2 & 8:00 & 252,85 & 252,26 \\
\hline & $05 / 11 / 14$ & 109,31 & 22,51 & 25,22 & 9:00 & 194,31 & 165,76 \\
\hline & $05 / 11 / 14$ & 313,27 & 22,37 & 34 & $10: 00$ & 453,19 & 613,74 \\
\hline & $05 / 11 / 14$ & 481,65 & 23,3 & 36,43 & $11: 00$ & 706,28 & 875,69 \\
\hline & $05 / 11 / 14$ & 1011,43 & 29,45 & 41,76 & $12: 00$ & 1562,09 & 1499,80 \\
\hline & $05 / 11 / 14$ & 988,45 & 29,31 & 49,84 & $13: 00$ & 1531,05 & 1463,63 \\
\hline & $05 / 11 / 14$ & 988,07 & 31,82 & 46,54 & $14: 00$ & 1464,87 & 1286,60 \\
\hline & $05 / 11 / 14$ & 832,56 & 29,6 & 48,26 & $15: 00$ & 1191,59 & 1253.2 \\
\hline & $05 / 11 / 14$ & 580 & 29,73 & 41,11 & $16: 00$ & 752,27 & 831,28 \\
\hline & $05 / 11 / 14$ & 98,97 & 26,43 & 30,27 & $17: 00$ & 154,03 & 155.29 \\
\hline & $05 / 11 / 14$ & 28,33 & 24,98 & 26,66 & $18: 00$ & 36,72 & 27,03 \\
\hline
\end{tabular}

Fonte: Elaborado pelos autores

A Figura 7 mostra os valores de potência medidos e simulados, (Vide Tabela 4), conforme a rede mais adequada de 25 neurônios para o algoritmo de LevenbergMarquardt. A linha contínua mostra valores de potência em Watts (W) medidos no sistema fotovoltaico em estudo. Estes valores foram utilizados para treinar a rede neural. A linha pontilhada mostra os valores obtidos após simulação da rede que se mostrou mais adequados para este tipo de análise. Observa-se neste comparativo que os valores ficam muito próximos, dentro dos limites de erro aceitáveis.

Figura 7 - Comparativo de potência medida (Target) usado para treinar a rede com a potência simulada

Potência Medida (target) e Simulada para um dia da estação Primavera (2014)

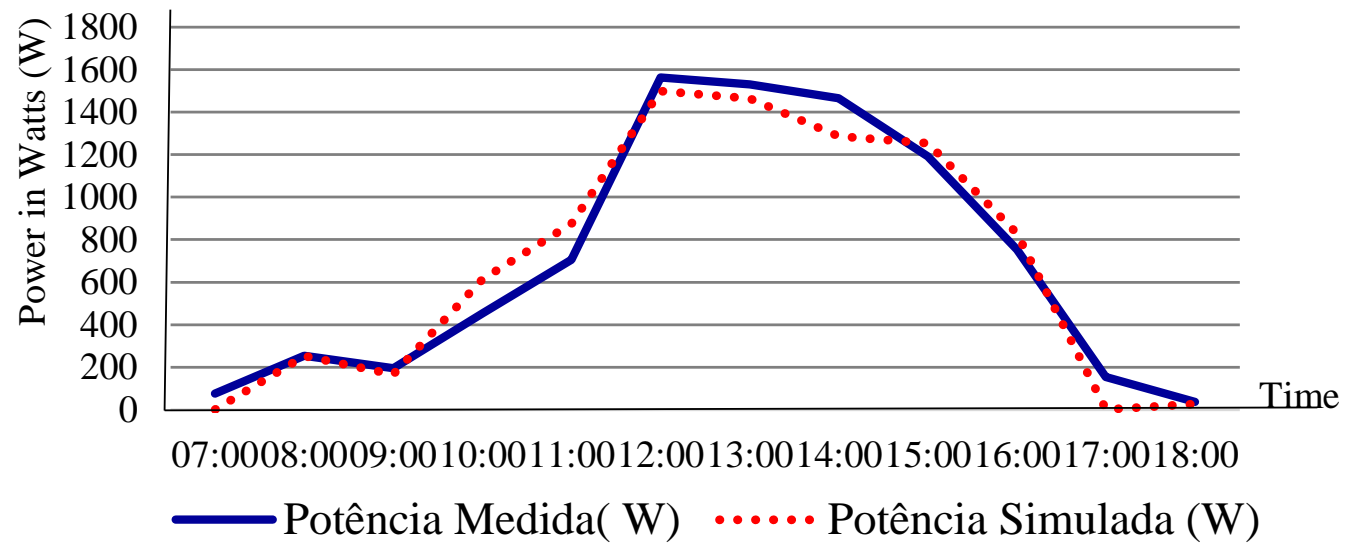

Revista Produção Online. Florianópolis, SC, v.17, n. 4, p. 1204-1217, 2017. 


\section{CONCLUSÕES}

Com o desenvolvimento de redes neurais artificiais buscou-se avaliar o ajuste do algoritmo Levenberg-Marquardt para análise de geração de energia elétrica de um sistema fotovoltaico, integrado a uma edificação na Universidade Federal de Santa Catarina, em Florianópolis. As redes foram construídas com arquitetura de uma camada de entrada com quatro variáveis e uma camada escondida com 5, 10, 15, 20, 25, 30 e 60 neurônios. A função de transferência foi a log Sigmóide e a de saída foi a purelin. Considerando os valores de entrada irradiação incidente na mesma inclinação dos módulos fotovoltaicos, temperatura ambiente, temperatura dos módulos e horários, comprovou-se que as redes propostas são capazes de realizar adequadas análises de potência e que no presente estudo atingiram correlações superiores a 0,98 , destacando-se a rede com 25 neurônios na camada escondida. Conforme resultados obtidos, conclui-se que este método LevenbergMarquardt é adequado para análise de potência de um sistema fotovoltaico.

\section{REFERÊNCIAS}

BOUSFIELD, P. M. Uso de uma rede neural para a previsão do volume de lodo gerado em estações de tratamento de efluente: um estudo de caso, 2008. Universidade da região de Joinville. Disponível em:<file:///C:/Users/Usuario/Dropbox/DOUTORADO/TESE/Referencias/REDES NEURAIS ARTIFICIAIS/Dissertacao Paulo Marcondes Bousfield.pdf>. .

CHATZIAGORAKIS.P., GEORGOULAS. PAPADOPOULOU, S. ELMASIDES,C., GIAOURIS, D., Seferlis, P., Sirakoulis, CH.G., KARAFYLLIDIS, I., PAPADOPOULOS, A. I., STERGIOPOULOS, F., ZIOGOU, C., IPSAKIS, D., Voutetakis, S. e ANDREADIS, I. Application of Neural Networks Solar Radiation Prediction for Hybrid Renewable Energy Systems. Engineering Applications of Neural Networks, v. 459, p. 133-144, 2014.

CHOW, S. K. H.; LEE, E. W. M.; LI, D. H. W. Short-term prediction of photovoltaic energy generation by intelligent approach. Energy and Buildings, v. 55, p. 660-667, 2012. Elsevier B.V. Disponível em: <http://linkinghub.elsevier.com/retrieve/pii/S037877881200415X>. https://doi.org/10.1016/j.enbuild.2012.08.011

FIORIN, D. V; MARTINS, F. R.; SCHUCH, N. J. Aplicacões de redes neurais e previsões de disponibilidade de recursos energéticos solares. Revista Brasileira de Ensino de Física, v. 33, n. 1, p. 13091 - 1309 20, 2011. http://dx.doi.org/10.1590/S1806-11172011000100009

GILMAN, A. Dobos; KASBERG, M. P50/P90. Analysis for solar energy systems using the system advisor model, NREL, May 13-17, 2012. Disponível em:< http://www.nrel.gov/docs/fy12osti/54488.pdf>. 
HAGAN, Martin T.; DEMUTH, Howard B.; BEALE, Mark Hudson; JESÚS, Orlando De. Neural network design. 2. ed. eBook, 2014.

MEKKI, A., ALOUI, F., DHOUIB, A., SAYADI, S. Effects of Phanerochaete chrysosporium on biologic activity of soil amended with olive mill wastewaters. Journal of Soil Science and Environmental Management, v. 3, n.1, p. 1-8, 2012. DOI: 10.5897/JSSEM11.092.

MELLIT, A.; KALOGIROU, S. A.; HONTORIA, L.; SHAARI, S. Artificial intelligence techniques for sizing photovoltaic systems: a review. Renewable and Sustainable Energy Reviews, v. 13, n. 2, p. 406-419, 2009. Disponível em:

<http://linkinghub.elsevier.com/retrieve/pii/S1364032108000051>. DOl: 10.1016/i.rser.2008.01.006

MELLIT, A.; KALOGIROU, S. A. ANFIS-based modelling for photovoltaic power supply system: A case study. Renewable Energy, v. 36, n. 1, p. 250-258, 2011. Elsevier Ltd. Disponível em:<http://linkinghub.elsevier.com/retrieve/pii/S0960148110002843>. DOI: 10.1016/..renene.2010.06.028.

MELLIT, A.; KALOGIROU, S. A. Artificial intelligence techniques for photovoltaic applications: a review. Progress in Energy and Combustion Science, v. 34, n. 5, p. 574632, 2008. https://doi.org/10.1016/j.pecs.2008.01.001

NREL. National renewable energy laboratory: research cell efficiency records. 2016. Disponível em: <http://www.nrel.gov/ncpv/>. Acesso em: junho de 2016.

RÜTHER, R. e DACOREGIO, M., 2000. Performance assessment of a 2 kwp gridconnected, building-integrated, amorphous silicon photovoltaic installation in Brazil. Prog.

Photovolt. Res. Appl. n. 8, p. 257 - 266. DOI: 10.1002/(SICl)1099159X(200003/04)8:2<257::AID-PIP292>3.0.CO;2-P

RÜTHER, R., 1998. Experiences and operational results of the first grid-connected, buildingintegrated, thin-film photovoltaic installation in Brazil. WCPEC2 - 2nd World Conference on Photovoltaic Solar Energy Conversion. Vienna, Austria.

YADAV, A. K.; CHANDEL, S. . Artificial Neural Network based Prediction of Solar Radiation for Indian Stations. International Journal of Computer Applications, v. 50, n. 9, p. 1-4, 2012. DOI:10.5120/7796-0907.

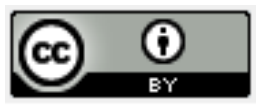

Artigo recebido em 31/07/2016 e aceito para publicação em 10/10/2017

DOI: http://dx.doi.org/10.14488/1676-1901.v17i4.2542 